\title{
Anemia lurking in introns
}

\author{
Narla Mohandas
}

New York Blood Center, New York, New York, USA.

\begin{abstract}
Anemia is defined by low levels of circulating hemoglobin, resulting in insufficient tissue oxygenation. This condition results from both genetic and nutritional factors and affects more than a billion people worldwide. For the inherited anemias, progress made over the last $\mathbf{4 0}$ years has increased our understanding of the structural basis for normal red cell membrane function and allowed definition of the genetic and pathophysiological bases of many human RBC membrane disorders. Despite these advances, there are continued uncertainties in the genotype-phenotype relationship in cases of severe, membrane-linked anemia. In this issue of the $J C I$, Gallagher and colleagues have identified a severe form of inherited anemia that results from aberrant splicing of $\alpha$-spectrin, which in turn leads to abnormal erythrocyte membrane structure and function. The identification and characterization of this splicing-associated genetic disease will facilitate diagnosis and treatment of severe anemia in affected patients. These findings not only improve understanding of red cell disorders, they are likely to impact many disciplines, as the disease-associated alternate branch point utilization defined in the report may be the underlying etiology for many other inherited or acquired disorders.
\end{abstract}

complex between the spectrin dimer, actin, and protein $4.1 \mathrm{R}$ is assembled at the other end of spectrin, and this spectrin-actinprotein 4.1R protein complex is also a key regulator of membrane mechanical stability. Decreased membrane mechanical stability (Figure 1) leads to cell fragmentation during the circulatory life span, with resultant increases in cell sphericity, which promotes splenic sequestration of the fragmented red cells.

\section{Inherited red cell membrane disorders}

The two most common inherited red cell membrane disorders are hereditary spherocytosis (HS) and hereditary elliptocytosis (HE), which lead to variable degrees of anemia (2-4). HS is the most common inherited hemolytic anemia in people of Northern European ancestry, although people of every ethnic background are affected. Inheritance is autosomal dominant in approximately two-thirds of cases, while autosomal recessive inheritance or de novo mutations account for the remaining cases. Mutations that lead to decreased membrane content of ankyrin, such as those in band $3, \alpha$ - and $\beta$-spectrin, and protein 4.2 , and subsequent loss of membrane cohesion results in HS (2). As described by Peter Agre in a series of papers (5-8), clinically, patients with recessive inherited HS (rHS) are more severely affected than patients with typical, dominant HS. Many rHS patients present with life-threatening hemolytic anemia in infancy or early childhood, and some rHS patients are transfusion-dependent for life. Erythrocytes from most rHS patients are spectrin deficient, and the degree of spectrin deficiency correlates with the degree of hemolysis and clinical response to splenectomy. The mechanisms of spectrin deficiency in most cases of rHS, until the report by Gallagher et al. in this issue, have not been defined.

$\mathrm{HE}$, another common inherited hemolytic anemia, is also found in every ethnic background but is particularly prevalent in malaria endemic regions of West Africa. Mutations in $\alpha$ - and $\beta$-spectrin and pro- 


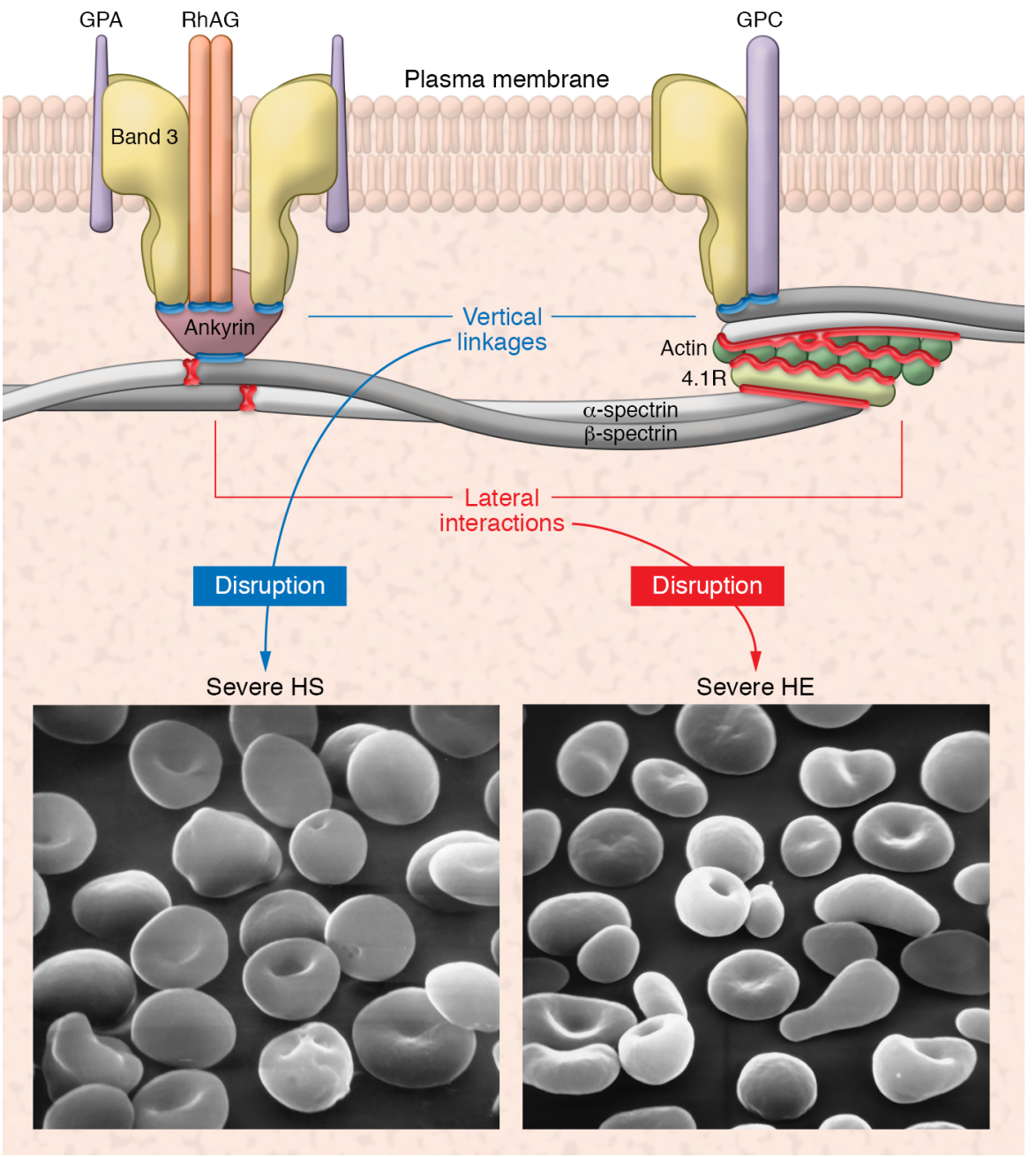

Figure 1. Key red cell membrane proteins regulating membrane cohesion and mechanical stability. Simplified schematic representation of the structural organization of key red cell membrane proteins that regulate membrane cohesion and membrane mechanical stability (top). Decreased numbers of vertical linkages leads to HS, and weakening of lateral interactions leads to HE. In both HS and HE, additional genetic changes that result in quantitative spectrin deficiency lead to a severe anemic phenotype with marked alterations in red cell morphology (bottom). Original magnification, $\times 4250$.

tein 4.1 that weaken lateral interactions among skeletal proteins lead to decreased membrane mechanical stability and the resultant elliptocytic morphology and membrane fragmentation. While HEassociated anemia is generally mild to moderate, a subset of patients with $\mathrm{HE}$ exhibit severe anemia characterized by erythrocytes that are abnormally sensitive to heat and have morphology similar to that seen in patients with thermal burns (9). The genetics of hereditary pyropoikilocytosis (HPP) suggest that patients with this disorder usually fall into one of three categories: (a) those who are homozygous for a structural variant of spectrin, typically in the region of spectrin self- association, (b) those who may be compound heterozygous for structural variants of the self-association site, and finally, (c) those who may be heterozygous for a single structural variant of spectrin self-association and who possess a second, uncharacterized defect $(2,10,11)$. The last group of patients exhibits marked spectrin deficiency, suggesting the presence of a production-defective or a thalassemia-like $\alpha$-spectrin allele in trans with the structural variant. The parent who transmits the production-defect allele is clinically and biochemically normal. As with rHS, the mechanisms of spectrin deficiency in HPP, until the report by Gallagher and colleagues, has not been defined.

\section{Mechanistic insights into spectrin-deficiency in HS and HPP}

Gallagher et al. utilized whole-exome sequencing (WES) to analyze the DNA from 24 kindreds with rHS, HPP, or undiagnosed transfusion-dependent anemia and identified numerous mutations in genes encoding erythrocyte membrane $\alpha$-spectrin (SPTA1) (12). Twenty-eight of the identified mutations were novel, and null alleles were found in trans to missense mutations. Interestingly, while a third of alleles (17/48) had no mutations, a rare intron 30 variant in SPTA 1 was seen in all 17 mutation-negative alleles. Bioinformatic analyses suggested this rare intron variant activates an alternate branch point (BP), a critical element of normal mRNA splicing. Minigene and CRISPR/Cas9-based gene editing studies revealed that the intron 30 variant changes a weak alternate $\mathrm{BP}$ to a strong $\mathrm{BP}$ in the context of a poor primary BP. This change leads to increased utilization of an alternate 3 ' splice acceptor site, creating an elongated mRNA transcript that causes a frame shift during protein synthesis, leading to spectrin deficiency. Inhibition and minigene studies demonstrated that the newly created termination codon activates nonsense-mediated decay, accounting for spectrin deficiency.

\section{Therapeutic implications and conclusions}

In most symptomatic cases of inherited hemolytic anemia due to disorders of the erythrocyte membrane, splenectomy is curative. However, in some patients with rHS and HPP, splenectomy is only palliative, as the need for transfusion in these patients with severe anemia is decreased, but not eliminated. The identification of a splicing-associated cause for spectrin deficiency raises a potential new target for therapeutic manipulation using genebased strategies to alleviate severe anemia.

The reported findings of Gallagher et al. represent a significant advance in our mechanistic understanding of severe anemia in human red cell membrane disorders. Exome sequencing and wholegenome sequencing are very helpful for defining the molecular basis for human diseases in well-characterized protein coding and RNA splice junction regions. However, the present study shows that 
other less well-studied regions, such as splice BPs and splicing enhancers, must also be examined. Importantly, the reported observations are likely to have much broader impact across many disciplines, as alternate BP utilization may be the underlying etiology of other inherited or acquired human disorders. Finally, the findings of Gallagher and colleagues suggest potential therapeutic targets for genebased and other therapeutic strategies in these human disorders.

Address correspondence to: Narla Mohandas, New York Blood Center, 310 East 67th Street, New York, New York 10065, USA. Phone: 212.570.3056; Email: mnarla@ nybc.org.
1. Mohandas N, Gallagher PG. Red cell membrane: past, present, and future. Blood. 2008;112(10):3939-3948.

2. Mohandas N. Inherited hemolytic anemia: a possessive beginner's guide. Hematology Am Soc Hematol Educ Program. 2018;2018(1):377-381.

3. Perrotta S, Gallagher PG, Mohandas N. Hereditary spherocytosis. Lancet. 2008;372(9647):1411-1426.

4. Gallagher PG. Hereditary elliptocytosis: spectrin and protein 4.1R. Semin Hematol. 2004;41(2):142-164.

5. Agre P, Orringer EP, Bennett V. Deficient red-cell spectrin in severe, recessively inherited spherocytosis. N EnglJ Med.1982;306(19):1155-1161.

6. Agre P, Casella JF, Zinkham WH, McMillan C, Bennett V. Partial deficiency of erythrocyte spectrin in hereditary spherocytosis. Nature. 1985;314(6009):380-383.

7. Chasis JA, Agre P, Mohandas N. Decreased membrane mechanical stability and in vivo loss of surface area reflect spectrin deficien- cies in hereditary spherocytosis. J Clin Invest. 1988;82(2):617-623.

8. Agre P, Asimos A, Casella JF, McMillan C. Inheritance pattern and clinical response to splenectomy as a reflection of erythrocyte spectrin deficiency in hereditary spherocytosis. N Engl J Med. 1986;315(25):1579-1583.

9. Zarkowsky HS, Mohandas N, Speaker CB, Shohet SB. A congenital haemolytic anaemia with thermal sensitivity of the erythrocyte membrane. Br J Haematol. 1975;29(4):537-543.

10. Wichterle H, Hanspal M, Palek J, Jarolim P. Combination of two mutant alpha spectrin alleles underlies a severe spherocytic hemolytic anemia. J Clin Invest. 1996;98(10):2300-2307.

11.Hanspal M, Hanspal JS, Sahr KE, Fibach E, Nachman J, Palek J. Molecular basis of spectrin deficiency in hereditary pyropoikilocytosis. Blood. 1993;82(5):1652-1660.

12.Gallagher PG, et al. Aberrant splicing contributes to severe $\alpha$-spectrin-linked congenital hemolytic anemia. J Clin Invest. 2019;129(7):2878-2887. 